\title{
Measuring the unmeasurable
}

\author{
After more than a century, the reality of Maxwell's displacement current has been demonstrated by \\ direct measurement. So, too, but less conclusively, has been the Aharonov/Bohm effect.
}

ONE of the big surprises of Maxwell's theory of electromagnetism was his introduction of the concept of displacement current. By Maxwell's time, it was generally understood that an electrical current in a conductor would generate a magnetic field (Ampère's law applies). In modern language, the relationship is that the curl of $\mathbf{H}$, the magnetic field strength, is equal to the current, or that $\nabla \times \mathbf{H}=\mathbf{J}$, where $\mathbf{H}$ is the field and $\mathbf{J}$ the current, both of them vector quantities. Maxwell's innovation was to add to the right-hand side of the equation the rate of change with time of the electrical displacement, $\mathbf{D}$, related to the electric field strength by $\mathbf{D}=\epsilon \mathbf{E}$, where $\epsilon$ is the scalar permittivity of the material, itself related to the dielectric constant by a numerical factor depending only on the choice of units. Now the displacement current has been measured for what appears to be the first time.

None of this implies that the reality of the displacement current has, been in limbo in the interval. Indeed, were it not for the displacement current, it would not be possible to deduce from Maxwell's equations that electromagnetic waves have the properties of light. Maxwell was well aware of the difficulty he was creating. Indeed, D.F. Bartlett and T.R. Corle from the University of Colorado at Boulder, who have now measured a displacement current directly (Phys. Rev. Lett. 55, $59 ; 1985)$, quote him as describing as one of the "chief peculiarities" of his thesis "that the time derivative of the electric displacement must be taken into account".

The principle of the measurement is a good deal simpler than the practice. The obvious first step is to create a reasonable source of displacement current. What could be simpler than the steady increase of electrical displacement between the plates of a capacitor during the process of being charged, most simply by connecting its plates to a physical electric current?

Bartlett and Corle point out two ob vious snags. First, the voltage would quickly reach the point at which there would be a spark between the capacitor plates. Second, since the obvious way of measuring the displacement current is to measure the associated magnetic field, the feasibility of the measurement is deter mined by the relative magnitude of the field and the sensitivity of available measuring devices. Bartlett and Corle point out that for realistic capacitors, the expected magnitude of the magnetic field will be measured in micro-gauss.

From there, the design of the measurement is determined. The ideal of a directcurrent measurement is unattainable, but a low-frequency current will do instead provided that the response-time of the ultimate measuring device is faster than the oscillation. The other requirement, that it should be possible to measure magnetic fields in the micro-gauss region, points uniquely to a SQUID (for superconducting quantum interference detec tor). And that, inevitably, points to the source of the complexity of the measurement, the need to contain the equipment in liquid helium.

Bartlett and Corle use a capacitor consisting of a pair of circular plates $7.6 \mathrm{~cm}$ in diameter and separated by $1.22 \mathrm{~cm}$. The magnetic field between them is measured by a coil $1.5 \mathrm{~mm}$ in diameter encased in a stainless steel tube which, at liquid helium temperatures, acts as a shield against stray electric fields. The SQUID itself, based on a Josephson junction, is enclosed in a superconducting lead shield. If the arrangement were strictly symmetrical, the magnetic field induced by the displacement current, at some given phase of the driving current, would be represented by concentric circular lines of force with the field strength increasing linearly from the centre to the edge of the plates and then decreasing inversely with distance from the axis of symmetry.

At least to a first approximation, the geometry is exactly like that of the magnetic field associated with a current in a linear conductor except that, now, the current is displacement current and distributed across the whole area of the plates.

The complications are horrendous. Magnetic fields from the cables carrying the current are an obvious nuisance, if only because the measurement must be made inside a liquid-helium Dewar vessel and also inside a shield against stray magnetic fields from elsewhere. (The shield is a spherical copper vessel coated on the inside by superconducting lead/tin solder and filled with helium.) The leads carrying current to the plates are bent so that their final separate approach to the capacitor is along the axis of the arrangement, and also that the magnetic field generated by the power supply can be measured simply by rotating the measuring coil through 90 degrees. The use of an alternating power supply has the advan- tage of allowing the phase of the oscillation of the displacement current to be determined directly.

The only surprise is the precision of the result. A plot of the field strength against distance from the centre of the plates is indeed a straight line, whose stope is determined by the small scatter of points to within three parts in 100 . The measured slope differs from that calculated from the dimensions of the capacitor and the other parameters of the measurement by less than five per cent, possibly explained by the intervention of a steel tube between the two plates.

Not all physical measurements of this kind are so unsurprising, demonstrations as it were of the ingenuity of which physicists are capable. A few weeks ago (Phys. Rev. Lett. 54, 2469; 1985), Giorgio Matteucci and Guilo Pozzi from the University of Bologna reported a measurement designed to verify the reality of the Aharonov/Bohm effect, the quantum phenomenon by means of which the phase of the wave function describing a particle (such as an electron) should be affected by the electromagnetic potential of an electromagnetic field even when the particles are not subjected to physical forces.

One of the first demonstrations of the effect, in which Matteucci and Pozzi also had a hand, consists in arranging that a beam of electrons will travel on either side of a superconducting tube with a magnetic field along its axis in such a way that magnetic forces do not affect the electrons. Although the apparent physical tracks of the electrons are unchanged, their phases are altered by the presence of the confined magnetic field, and interference fringes produced as a result

What Matteucci and Pozzi have now done is to repeat the experiment with a thin platinum wire coated on one side only with a thin layer of aluminium. Because of the contact potential between the two metals, the electrons see the wire as if it were an unsymmetrical distribution of electric charge, but because the wire is thin, there is no effective force on the electrons passing on one side or the other. But yet again, because in this case the electrostatic potential is different on one side of the wire from the other, the phases change and electron interference fringes are produced. Indeed, the pattern of the fringes can be changed as the wire is rotated about its axis which will seem yet another tour de force.

John Maddox 